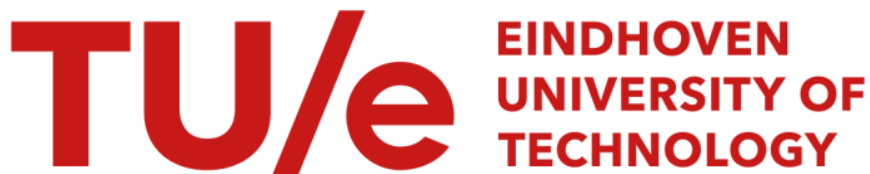

\section{On the use of focal-plane arrays in $\mathrm{mm}$-wave $5 \mathrm{G}$ base stations}

Citation for published version (APA):

Elsakka, A., Bressner, T. A. H., van den Biggelaar, A. J., Al-Rawi, A., Johannsen, U., Ivashina, M. V., \& Smolders, A. B. (2018). On the use of focal-plane arrays in mm-wave 5G base stations. In 12th European Conference on Antennas and Propagation (EUCAP 2018) Institution of Engineering and Technology (IET). https://doi.org/10.1049/cp.2018.1207

DOI:

10.1049/cp.2018.1207

Document status and date:

Published: 10/12/2018

\section{Document Version:}

Accepted manuscript including changes made at the peer-review stage

\section{Please check the document version of this publication:}

- A submitted manuscript is the version of the article upon submission and before peer-review. There can be important differences between the submitted version and the official published version of record. People interested in the research are advised to contact the author for the final version of the publication, or visit the $\mathrm{DOI}$ to the publisher's website.

- The final author version and the galley proof are versions of the publication after peer review.

- The final published version features the final layout of the paper including the volume, issue and page numbers.

Link to publication

\section{General rights}

Copyright and moral rights for the publications made accessible in the public portal are retained by the authors and/or other copyright owners and it is a condition of accessing publications that users recognise and abide by the legal requirements associated with these rights.

- Users may download and print one copy of any publication from the public portal for the purpose of private study or research.

- You may not further distribute the material or use it for any profit-making activity or commercial gain

- You may freely distribute the URL identifying the publication in the public portal.

If the publication is distributed under the terms of Article 25fa of the Dutch Copyright Act, indicated by the "Taverne" license above, please follow below link for the End User Agreement:

www.tue.nl/taverne

Take down policy

If you believe that this document breaches copyright please contact us at:

openaccess@tue.nl

providing details and we will investigate your claim. 


\title{
On the Use of Focal-Plane Arrays in mm-Wave 5G Base Stations
}

\author{
A. Elsakka, T.A.H. Bressner, A.J. van den Biggelaar, A. Al-Rawi, U. Johannsen, M.V. Ivashina, A.B. Smolders \\ Electromagnetics Group, Eindhoven University of Technology (TU/e) \\ Eindhoven, The Netherlands \\ a.a.h.m.elsakka@tue.nl
}

\begin{abstract}
In mobile communications, the research and development for the fifth generation (5G) has gained significant interest in the recent years. The high data rate requirements for $5 G$ introduce more challenges in terms of the system power consumption. Hence, considering the base station, a more efficient antenna system is necessary in order to provide higher signal to noise ratios to achieve the required data rates, while consuming the lowest possible amount of power. In this paper a phased array fed reflector is proposed as a solution for $5 \mathrm{G}$ base stations. The main idea is to use the high gain of a reflector antenna to achieve high effective isotropic radiated power (EIRP). That in turn allows the reduction of the number of feed array elements as well as the amount of supplied power. Consequently, the low cost silicon-based amplifiers can be utilized . The proposed solution includes our vision about some aspects of the antenna system regarding cell coverage, beamforming as well as antenna gain and EIRP. Finally, a system concept for the base station antenna subsystem is presented.
\end{abstract}

Index Terms-Array fed reflector, phased arrays, reflector antenna, focal plane arrays, $\mathrm{mm}$-wave.

\section{INTRODUCTION}

$\mathbf{T}$ HE rapid growth in mobile applications urges a rapid increase in the data rates and overall system capacity, which will be one of the main motives for fifth generation (5G) and beyond in mobile technology. Traditionally, in early days of cellular mobile systems, base station antennas were omni-directional antennas having a cylindrical shape [1]. In order to fulfill the market demands, more advanced antenna solutions were introduced through time to achieve higher system capacity and efficient use of the frequency band. Accordingly, panel antennas were introduced. They are composed of a small number of antenna array elements in front of a ground plane. This antenna usually covers an azimuth range of $\pm 60^{\circ}$ or $\pm 30^{\circ}$, resulting into dividing the cell area into three or six sectors respectively. Through mobile generations more advancements were made to that antenna configuration such as introducing dual polarization, multi-bands, and even a narrow usage of a few number of multi-beam solutions as explained in [1].

Moving to $5 \mathrm{G}$, a target peak data rate of $10 \mathrm{Gbps}$ is anticipated [2]. In order to fulfill that requirement, further developments are necessary to be applied to the base station. First, a larger frequency bandwidth; and hence allocating the communications channel frequency band in the millimeter waves (mm-waves) spectrum is necessary. The main challenge of mm-wave spectrum is the free-space path loss that increases significantly. Hence, in order to achieve a sufficient communication distance, the base station must be able to generate a high effective isotropic radiated power (EIRP) at mm-wave frequencies. Another required development is utilizing the massive multiple-input multiple-output (MassiveMIMO) concept, where the number of antennas in base station is greater than the number of users. That concept allows multi-beam generation and steering, separating users spatially and even sending multiple data streams to the same user. Furthermore, the massive MIMO technique simplifies the signal processing part of the system by creating "channel hardening" which in turn suppresses the small scale fading [3]. Moreover, the high beamforming gain eases the requirements of the transmitting power amplifiers leading to lower power consumption. However, the large number of antennas poses a challenge with respect to using fully digital beamforming, as the number of the analog to digital converters (ADC) and the digital to analog converters (DAC) will be huge which again will lead to high power consumption and the need for active cooling of the front-end [4]. Here, the use of a hybrid beamformer can be a promising solution to that problem. Finally, above mentioned features have to be achieved while reducing the overall system cost to suite the market needs.

In order to meet those demands, a phased array antenna system is a commonly considered and examined concept [4], [5]. This antenna system has the advantage of flexibility and high EIRP that scales to $N^{2}$, where $N$ is the number of antenna elements. However, the heat dissipation due to the huge power consumed and small spacing between antenna elements would be an obstacle. And increasing the antenna elements spacing will lead to the appearance of grating lobes in case of linear arrays. Furthermore, when $n$ user equipments (UE) are served at the same time, the transmit power will be divided between them which leads to lower EIRP, and hence, a lower data rate. Finally, the transmit power of the UE is much less than what can be provided by the base station, which leads to lower EIRP in the uplink. To compensate for the lower EIRP in the uplink with a phased array antenna, more antenna elements need to be combined in order to produce high gain. Thus, the phased array antenna may not be a cost effective solution.

Another promising solution is a phased array fed (PAF) reflector antenna [6]. The passive reflector will be responsible for producing a high gain to increase the EIRP, in contrast to the traditional phased array case where the beamformer utilizes large number of antennas to produce high antenna gain. PAF approach leads to a reduction of the number of antenna elements, which in turn helps to overcome the heat dissipation problem. This solution has been proposed and 
successfully implemented in modern radio telescopes [7]. This radio telescope employed large scale (massive) receiving antenna array as a feed for a reflector enabling a cost effective solution with respect to directly radiating phased arrays. Furthermore, reducing the number of antenna elements may allow utilizing a fully digital beamformer, which has the advantage of high flexibility. Moreover, the high antenna gain will compensate the low received power in the uplink leading to a better bi-directional communication. While multiple beam generation and steering will be achieved by illuminating the reflector from different points on the reflector's focal plane. However, the scanning capabilities of reflector antennas are typically lower than that of directly radiating phased array antennas [8]. Hence, more complex design approaches need to be developed.

In this paper, the power consumption issue for $5 \mathrm{G}$ antenna systems is studied and several ways to tackle the problem using a PAF reflector antenna system are presented. In Section II the antenna requirements and design challenges from the perspectives of gain and coverage are discussed. While in Section III a system concept is introduced in order to provide an efficient antenna system that minimizes the power consumption. Finally, in Section IV the conclusion and future work are presented.

\section{ANTENNA REQUIREMENTS AND DESIGN CHALLENGES}

The antenna system requirements can be summarized as follows; firstly, generate and steer multiple beams with high EIRP. Secondly, provide full coverage to the whole cell area in order to be able to serve all UEs within the cell. These two requirements have to be achieved with minimum system power consumption. In the upcoming subsections we discuss how to achieve these goals.

\section{A. Antenna gain and EIRP}

In our study, a peak data rate of $1 \mathrm{Gbps}$ is considered based on [10]. The specified data rates are to be achieved for the outdoor-to-outdoor urban micro cell (UMi) scenario, which is described in [11] and [12]. The required EIRP for each distance away from the base station can be derived as follows; first, the required receiver sensitivity is calculated as in [13] and [14] in order to achieve the required data rate. That calculation was done assuming QPSK modulation scheme and $800 \mathrm{MHz}$ channel bandwidth. Then, we add the average NLOS path loss for street canyon UMi (SC-UMi) scenario at each distance, where the data was taken from [15]. The SC-UMi was chosen since it was the highest path loss exponent among all measured path loss scenarios in [15].

Our first attempt to reduce the power is by utilizing siliconbased amplifiers with $8 \mathrm{dBm}$ average power per each antenna element for transmission. For a planar phased array antenna system, the power and gain can be calculated as follows:

$$
\begin{aligned}
& P_{t o t}=P_{a m p}+10 \log _{10}(N)+\eta_{t a p}, \\
& G_{t o t}=G_{i n d}+10 \log _{10}(N)+\eta_{\text {rad }},
\end{aligned}
$$

where $P_{\text {tot }}[\mathrm{dBm}]$ is the transmit power generated by the array, $P_{a m p}[\mathrm{dBm}]$ is the average power of one amplifier, $N$ is the number of elements and $\eta_{t a p}[\mathrm{~dB}]$ is the tapering efficiency. In (2), $G_{t o t}[\mathrm{dBi}]$ is the gain of the array, $G_{\text {ind }}[\mathrm{dBi}]$ is the gain of one element, and $\eta_{\text {rad }}[\mathrm{dB}]$ is the radiation efficiency. Then the EIRP $[\mathrm{dBm}]$ is calculated as:

$$
E I R P=P_{t o t}+G_{t o t} .
$$

Considering an array composed of patch antenna elements of approximately $5 \mathrm{dBi}$ gain per element as in [16], and assuming an optimistic scenario where the tapering and radiation efficiencies are of $100 \%$; the required number of antenna elements in order to achieve the required EIRP values are listed in Table I.

TABLE I

REQUIRED EIRP AND NUMBER OF ANTENNA ELEMENTS IN ORDER TO ACHIEVE 1GBPS FOR DIFFERENT DISTANCES (FOR PHASED ARRAY CASE)

\begin{tabular}{|ccc|}
\hline Distance[m] & EIRP[dBm] & $\begin{array}{c}\text { Number of antenna } \\
\text { elements }\end{array}$ \\
\hline \hline $25-50$ & 41 & 25 \\
$50-100$ & 51 & 79 \\
$100-150$ & 57 & 158 \\
\hline
\end{tabular}

However, if PAF reflector antenna systems are considered, the number of amplifiers (number of antenna elements) needed to provide the required EIRP will be reduced significantly due to the large antenna gain provided by the reflector. Table II shows the required gain values when utilizing different number of amplifiers (and, thus, antenna elements) in order to achieve the required EIRP listed in Table I. The required gain values are calculated for the maximum distance for the outdoor-to-outdoor UMi scenario.

TABLE II

REQUIRED ANTENNA GAIN IN ORDER TO REACH MINIMUM AND MAXIMUM CELL DISTANCE USING DIFFERENT NUMBER OF ANTENNA ELEMENTS

\begin{tabular}{|ccc|}
\hline $\begin{array}{c}\text { Number of amplifiers } \\
\text { (antenna elements) }\end{array}$ & Avg. power [dBm] & $\begin{array}{c}\text { Antenna Gain[dBi] at } \\
\text { 150m (UMi) }\end{array}$ \\
\hline 1 & 8 & 49 \\
2 & 11 & 46 \\
4 & 14 & 43 \\
8 & 17 & 40 \\
16 & 20 & 37 \\
32 & 23 & 34 \\
64 & 26 & 31 \\
128 & 29 & 28 \\
\hline
\end{tabular}

The specified gains in Table II are achievable with a PAF reflector antenna such as in [9]. From this analysis it can be concluded that, if using a PAF reflector antenna, the number of antenna elements needed to reach a certain distance with the specified EIRP values can be reduced significantly compared to the phased array case. However, the number of antenna elements utilized depends also on other parameters such as the reflector focal field distribution and the used antenna array element. For example a parabolic reflector of $1 \mathrm{~m}$ diameter (D) and focal distance $(\mathrm{F})$ of $0.6 \mathrm{~m}$ is considered for analyzing the focal fields. The physical optics Matlab model utilized in [17] was used assuming $0^{\circ}$ angle of incidence. The estimated number of active elements in the focal fields was between 4 and 21 depending on the axial displacement towards the reflector. Based on this estimation, the requirements in Tables II and I are feasible. 


\section{B. Coverage and beamforming strategy}

For multi-beam antenna systems, there are two ways to provide coverage [18]:

1) Switched beam antenna systems; where the antenna provides a finite number of fixed beams (predefined pattern) to cover the whole area, see Fig. 1a. The UE switches between beams as it moves from one place to another, and the base station can turn off the idle beams.

2) Adaptive antenna systems; where the antenna provides multiple beams (scenario based, infinite number of patterns) that are able to freely track the user movement, as well as canceling any interfering signal, see Fig. $1 \mathrm{~b}$.

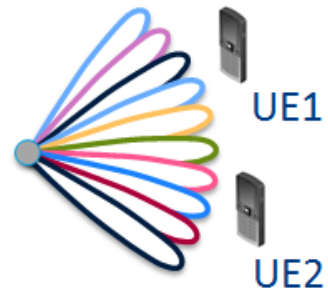

(a)

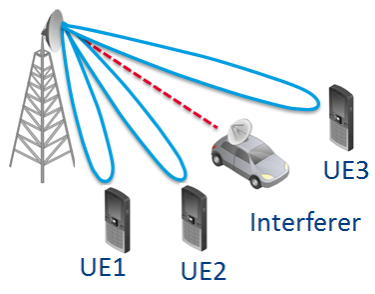

(b)
Fig. 1. (a) Switched beam configuration, and (b) adaptive beam configuration.

In the switched-beam approach, the antenna system provides fixed multi-beams pointing at different directions, covering the whole served area, see Fig. 1a. That approach has the advantage of dividing the served sector into smaller serving areas increasing the overall system capacity. It is also of low complexity and easy to implement by switching between antenna elements. However, the switched-beam approach does not guarantee that the served user is placed at the beam maximum. That may cause significant degradation in performance if an interferer was present at the beam maximum while the served user is located on a point of lower power level [18].

In order to cover the whole cell area simultaneously, a certain number of beams are needed. That number depends on the beam footprint, which depends on the beam width and the distance of the area covered by the beam away from the base station. This can be deduced using the equations in [19]. For shorter distances with respect to the base station, the area covered by a beam of certain HPBW becomes narrower compared to areas at longer distances. We consider two methods to provide coverage with switched beams:

- The first method employs muliple beams with variable HPBWs. Wide beams (lower gain) are provided for shorter distances and narrower beams (higher gain) for longer distances. This plan has the advantage of the small number of beams, and hence smaller number of utilized antenna elements. However, it has a degrading effect on the NLOS signals, as a signal can arrive from a far distance with large NLOS path loss, while it may be scattered by a scatterer close to the base station. Hence, it's direction of arrival will be where only a low antenna gain is provided, see Fig. 2.

- The second method employs multiple beams with constant narrow HPBW. Multi-beams with constant high gains are provided for near and far distances while covering the whole sector area. However, the high gain beams for shorter

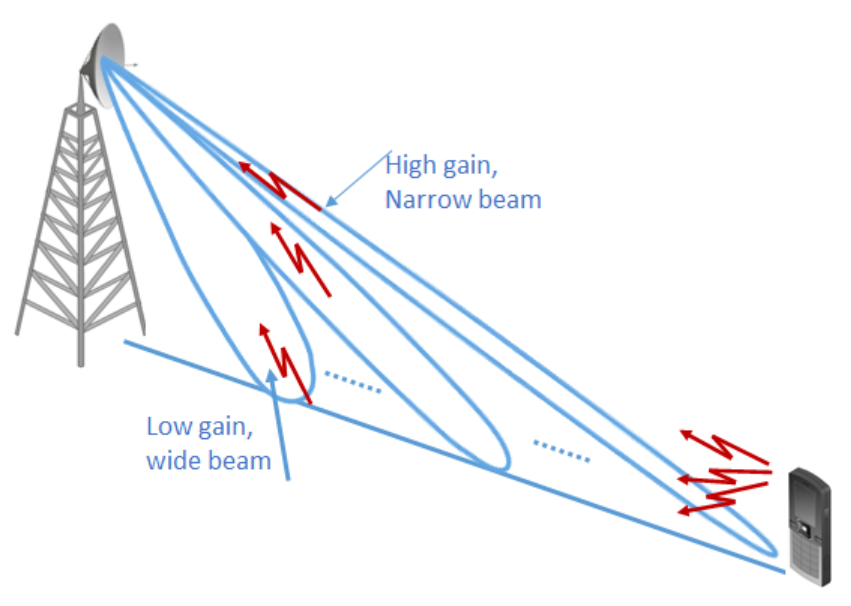

Fig. 2. Variable gain multiple beams

distances lead to losing coverage area due to the narrow HPBW. That leads to very short serving time by single beam for a moving user as well as huge number of beams for shorter distances, which means huge number of antenna elements. An estimation for the number of beams needed to cover the whole area is provided in Section III. The estimation states that around 255 beams are needed in the UMi scenario. As a result a large number of antenna elements are required. Thus, the switched-beam approach is not a promising concept to cover the entire cell and some adaptivity needs to be introduced.

The adaptive antenna system can be a promising solution. Here, the beams are not covering the whole sector area the whole time. Instead, the beams are only directed towards the signal coming from the UE [18]. This solution provides more flexibility and will lead to more efficient utilization of resources (antenna array elements). Furthermore, it can ensure the presence of the user at the point of beam maximum and nulls any signal coming from any interferer. However, this needs a fully digital beamformer which will lead to high power consumption.

Hybrid beamforming can reduce the power consumption and was, therefore, also adopted by 3GPP, see [20]. As mentioned in Section I, using a PAF reflector antenna system can allow a fully digital beamforming since the number of antenna elements will be limited. However, hybrid beamforming can also be applied here to reduce the power consumption even more.

\section{PROPOSED SySTEM CONCEPT}

In the proposed system concept, the cell area is divided into two regions (see Figs. 3a and $3 \mathrm{~b}$ ), each served by a different reflector antennas. The far region (i.e. $>>75 \mathrm{~m}$ ) is served by switched beams generated by high gain PAF reflector antenna. The antenna produce scanned beams with different angles by illuminating the reflector from different points on the focal plane. While for the closer region (i.e. $<<$ $75 \mathrm{~m}$ ) a cylindrical reflector will combine two concepts; focal line scanning in elevation, and adaptive phased array scanning is used in azimuth. This way we can overcome the poor reflector scanning capabilities and reduce the beamformer requirements. Furthermore, we ensure better serving time per beam for far distances since the beam footprint is sufficiently 
wide. In addition, since the UE moving with a certain speed has higher angular velocity for shorter distances, smooth UE tracking would be necessary and can be ensured through the digital beamforming. Moreover, the number of utilized antenna array elements can be reduced since the overall number of simultaneous beams is reduced. Finally, utilizing a hybrid beamformer (analog for far distance and digital for short distance as shown in Fig. 4) leads to the reduction of the system power consumption.

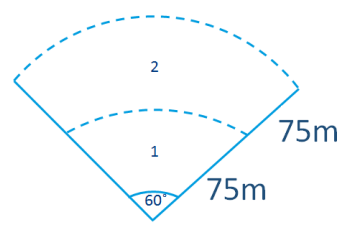

(a)

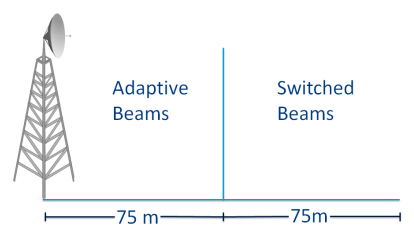

(b)
Fig. 3. Dividing the UMi cell area into two regions; (a) top view and (b) side view

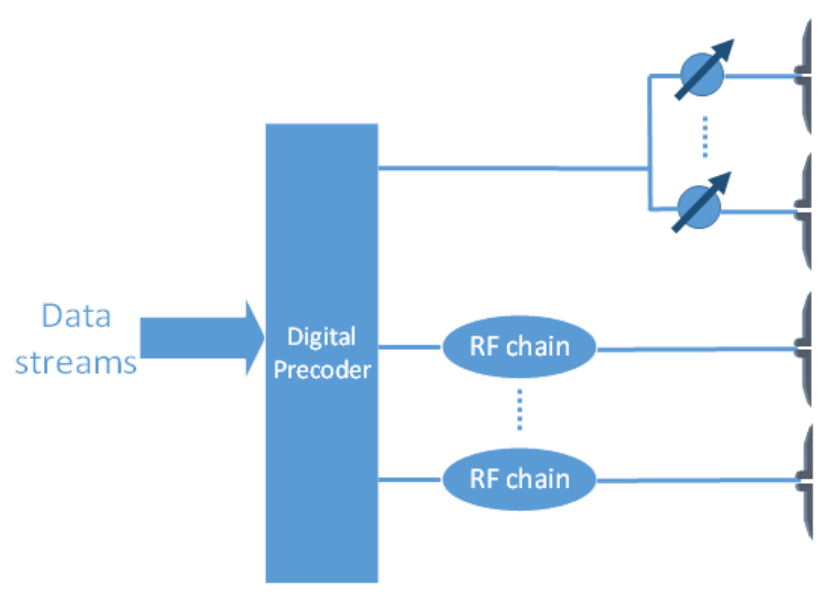

Fig. 4. Proposed hybrid beamforming architecture

To test our approach we use the equations in [19] to calculate beam areas at different distances and hence, the number of required beams can be computed. Assuming a HPBW of $1.65^{\circ}$ which corresponds to $40 \mathrm{dBi}$ gain, the approximate number of required beams for the far area would be 72 beams. On the other hand, in order to cover the full area simultaneously, around 255 beams will be required. That leads to relatively smaller number of utilized antenna elements to generate 72 simultaneous beams for far distance. And adaptively utilizing the rest of the array elements to generate beams according to UE presence. That in turn will lead to a reduction of the number of utilized antenna array elements and hence the antenna system will be more efficient.

\section{CONCLUSION}

To conclude, this paper presents a solution for $5 \mathrm{G}$ base stations using a PAF reflector antenna. The solution shows great potential to reduce the system power consumption by reducing the number of antenna elements and the power rating of the power amplifiers. However, due to the narrow beam width, a specialized beamforming strategy has to be developed. A system concept is presented that has the potential to reduce the number of antenna elements by dividing the sector area into two regions; one served with switching beam strategy and the other served by adaptive beam strategy.

\section{ACKNOWLEDGMENT}

This project has received funding from the European Unions Horizon 2020 research and innovation programme under the Marie Sklodowska-Curie grant agreement No 721732.

\section{REFERENCES}

[1] "MIMO and smart antennas for mobile broadband systems", 4 G Americas, Oct 2012.

[2] Ericsson white paper, "5G radio access," Uen 284 23-3204, Rev C Apr 2016. (2017,Jul)[Online] Available: https://www.ericsson.com/assets/ local/publications/white-papers/wp-5g.pdf

[3] Massive MIMO Properties based on Measured Channels: Channel Hardening, User Decorrelation and Channel Sparsity," in Signals, Systems and Computers, 2016 50th Asilomar Conference, Pacific Grove, CA, USA, Nov. 2016 .

[4] B. Sadhu et al, "7.2 A $28 \mathrm{GHz} 32$-element phased-array transceiver IC with concurrent dual polarized beams and 1.4 degree beam-steering resolution for 5G communication," in 2017 IEEE International SolidState Circuits Conference (ISSCC), pp 128-129, 2017

[5] A.B. Smolders, "Design and construction of a broadband wide-scan angle phased-array antenna with 4096 radiating elements," in Proceedings of International Symposium on Phased Array Systems and Technology, Boston, MA, pp. 87-92, 1996.

[6] (2017,Oct)[Online] Available: http://silika-project.eu/

[7] K.F. Warnick, R. Maaskant, M.V. Ivashina, D.B. Davidson, and B.D. Jeffs, "High Sensitivity Phased Array Receivers for Radio Astronomy," IEEE Proceedings, vol. 104, no. 3, pp. 607-622, May 2016.

[8] A. Dubok, A. Al-Rawi, N. Tessema, E. Tangdiongga, M.H.A.J. Herben, G. Gerini, and A.B. Smolders, "Double reflector configuration for optimal exposure of wideband focal plane arrays with optical beamforming," IEEE Trans. Antennas Propagat., vol. 65, no. 8, pp. 4316-4321, May 2017.

[9] B. Rohrdantz, T. Jaschke, T. Reuschel, S. Radzijewski, A. Sieganschin, and A. F. Jacob, "An Electronically Scannable Reflector Antenna Using a Planar Active Array Feed at Ka-Band," IEEE Trans. Microw. Theory Techn, vol. 65, no. 5, pp. 1650-1661, May 2017.

[10] ITU, "IMT Vision-Framework and overall objectives of the future development of IMT for 2020 and beyond," Tech. Rep. ITU-R M.2083-0, International Telecommunication Union, Geneva, Sept 2005.

[11] ITU, "Guidelines for evaluation of radio interface technologies for IMT Advanced," Tech. Rep. ITU-RM.2135-1, ITU, Geneva, Dec 2009.

[12] 3rd Generation Partnership Project; Technical Specification Group Radio Access Network, "Study on Scenarios and Requirements for Next Generation Access Technologies (Release 14)," Tech. Rep. 38.913 V14.0.0, 3GPP, France, Oct 2016.

[13] Q. Gu, "RF System Design of Transceivers for Wireless Communications", Springer US, 2006.

[14] M. Loy, "Understanding and Enhancing Sensitivity in Receivers for Wireless Applications," Tech. Rep. SWRA030, Texas Instruments Wireless Communication Business Uni, USA, May 1999.

[15] University Aalto, AT\&T, BUPT, CMCC, Ericsson, Huawei, Intel, KT Corporation, Nokia, NTT DOCOMO, New York University, Qualcomm, Samsung, University of Bristol, University of Southern California, "White paper: 5G Channel Model for bands up to $100 \mathrm{GHz}$ (Annex)," Tech. Rep. Annex, Department of Computer Science, Michigan State University, East Lansing, Michigan, September 2016.

[16] A.B. Smolders, R.M.C. Mestrom , A. Reniers and M. Geurts, "A Shared Aperture Dual-Frequency Circularly Polarized Microstrip Array Antenna," IEEE Antennas and Wireless Propagation Letters (AWPL), vol. 12, pp. 120-123, 2013.

[17] A. Al-Rawi, A. Dubok, S.J. Geluk, B.P. de Hon, M.H.A.J. Herben, and A.B. Smolders, "Increasing the EIRP by using FPA-fed reflector antennas," in Antennas and Propagation (APSURSI), Fajardo, Puerto Rico, USA, pp. 1623-1624, 2016.

[18] S. Bellofiore, C. A. Balanis, J. Foufz, and A. S. Spanias , "SmartAntenna Systems for Mobile Communication Networks Part I: Overview and Antenna Design ," IEEE Antennas and Propagation Magazine, vol. 44, no. 3, pp. 145-154, Jun. 2002.

[19] (2017,Jul)[Online] Available: https://www.allaboutcircuits.com/tools/ antenna-downtilt-and-coverage-calculator/

[20] 3GPP, "Final report of 3GPP TSG RAN WG1 no.85," 2016, available: http://www.3gpp.org 